American Journal of Applied Sciences 4 (9): 709-717, 2007

ISSN 1546-9239

(C) 2007 Science Publications

\title{
Determination of Metoprolol and Felodipine in Binary Mixture Using Chemometric- Assisted Spectrophotometric and High-Performance Liquid Chromatographic-UV Mmethods
}

\author{
${ }^{1}$ Hesham Salem and ${ }^{2}$ Ola M. Abdallah \\ ${ }^{1}$ Analytical Chemistry Department, Faculty of Pharmacy, Minia University, Minia, Egypt \\ ${ }^{2}$ Analytical chemistry department, Faculty of Pharmacy, Al-Azhar university, Cairo, Egypt
}

\begin{abstract}
Four methods were developed for simultaneous determination of metoprolol and felodipine without previous separation. In the first method both drugs were determined using first derivative UV spectrophotometry, with zero crossing measurement at 222 and $235 \mathrm{~nm}$ for metoprolol and felodipine, respectively. The second method depends on first derivative of the ratios spectra by measurements of the amplitudes at $250.1 \mathrm{~nm}$ for metoprolol and $224.3 \mathrm{~nm}$ for felodipine. Calibration graphs are established in the range of $20-150 \mu \mathrm{g} / \mathrm{ml}$ and $10-60 \mu \mathrm{g} / \mathrm{ml}$ for metoprolol and felodipine, respectively. The third method describes the use of multivariate spectrophotometric calibration for the simultaneous determination of the analyzed binary mixture where the resolution is accomplished by using partial least squares (PLS) regression analysis. Although the components show high degree of spectral overlap, they are simultaneously determined with high accuracy and without interference of pharmaceutical dosage form excipients. A comparison with the related multivariate method of classicial least squares (CLS) analysis is done showing less relative results due to severe spectral overlap of the studied drugs. In the fourth method (HPLC), a reversed-phase column and a mobile phase of methanol:water:acetonitrile $(70: 22: 8 \mathrm{v} / \mathrm{v} / \mathrm{v} /)$ at $0.9 \mathrm{ml} / \mathrm{min}$ flow rate is used to separate both drugs and UV detection at $260 \mathrm{~nm}$. Good linearities are obtained in concentration range of $0.15-15$ $\mu \mathrm{g} / \mathrm{ml}$ for metoprolol and $0.03-5 \mu \mathrm{g} / \mathrm{ml}$ for felodipine. All the proposed methods are extensively validated. They have the advantage of being economic and time saving. All the described methods can be readily utilized for the analysis of pharmaceutical formulations, the fourth method (HPLC) is successfully applied for the analysis of both drugs in human serum samples. The results obtained by adopting the proposed methods are statistically analyzed and compared with those obtained by reported methods.
\end{abstract}

Keywords: Metoprolol; Felodipine; First derivative spectrophotometry; Ratio derivative spectrophotometry; Multvariate; HPLC; Pharmaceuticals; Human serum

\section{INTRODUCTION}

Metoprolol is a beta-adrenoceptor blocking agent used for treatment of hypertension ${ }^{[1]}$. The most recent methods for determination of metoprolol included chromatographic $^{[2-5]}$, electrochemical ${ }^{[6,7]}$ and spectrophotometric ${ }^{[8-13]}$ techniques.

Felodipine is a vasodilator ${ }^{[1]}$. Several methods have been reported for felodipine assay including chromatographic ${ }^{[14-16]}$ and spectrophotometric ${ }^{[17-19]}$ techniques.

Metoprolol

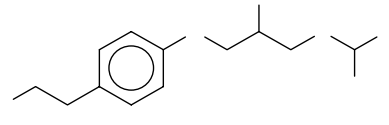

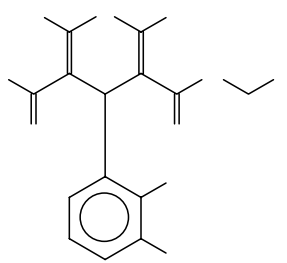

Felodipine

Recently, derivative spectrophotometry has been found to be a useful method in the determination of mixtures with two or more components having overlapping spectra and in eliminating interference from formulation matrix by using the zero-crossing techniques ${ }^{[20-22]}$.

Corresponding Author: Dr. H. Salem,Analytical Chemistry Department, Faculty of Pharmacy, MSA University, Cairo, Egypt 
Furthermore, ratio-spectra derivative spectrophotometric method has also been found to be useful in the estimation of drugs in their mixtures ${ }^{[23-27]}$. This method permits the determination of a component in their mixture at the wavelengths corresponding to a maximum or minimum and also the use of the peak-topeak between consecutive maximum and minimum. The main advantage of derivative of the ratio-spectra method may be the chance of easy measurements in correspondence of peaks so it permits the use of the wavelength of highest value of analytical signals (maximum or minimum). Moreover, the presence of a lot of maxima and minima is another advantage by the fact that these wavelengths give an opportunity for the determination of active compounds in the presence of other active compounds and excipients which possibly interfere the analysis.

Multivariate calibration methods applied to spectral data are being increasingly used for pharmaceutical analysis. Classical least squares ( CLS ) and Principal components regression (PCR) analysis are the most simplest multivariate methods that can be performed with easily accessible statistical software ${ }^{[28-31]}$.

CLS technique assumes that responses (absorbance) at each frequency (wavelengths) are proportional to component concentration units.

Model errors are assumed to derive from the measurement of spectral absorbance. So CLS requires that all interfering chemical components be known and included in the calibration data set. CLS has the advantage of improved precision when using many frequencies, due to signal averaging.

Calibration is realized by recording the spectra at $n$ wavelengths of $m$ standard mixtures, of known composition of $c$ components. The spectra (absorbance or emission) are arranged into the columns of matrix $Y$ (dimensions $n \times m$ ), with the composition of each mixture forming the columns of concentration matrix $X$ $(c \times m)$

$$
Y=K . X
$$

With a prior knowledge of $X$ and by recording data for $Y$, then the matrix of sensitivities, $K$, can be calculated, but after the rearrangement of equation 1 to the following equation by multiplying the equation components by $\mathrm{X}^{\mathrm{t}}$ value as:

$$
\begin{aligned}
& Y \cdot X^{t}=K \cdot X \cdot X \\
& K=\left(X \cdot X^{t}\right)^{-1} \cdot Y \cdot X^{t}
\end{aligned}
$$

To avoid being under-determined, there must be measurements at more wavelengths than there are components (i.e. $n \geq c$ ). If $n>c$ then the component concentrations in an unknown mixture are obtained from its spectrum by,

$$
X_{\text {unknown }}=\left(K^{t} \cdot K\right)^{1-} \cdot K^{t} y_{\text {unknown }}
$$

This CLS method is intuitively appealing since it is based on some generally assumed relationship, e.g. Beer's law, and it can be used for moderately complex composition of the calibration mixtures, i.e. the concentration of each absorbing species. PCR is a twostep procedure, in the first step, one estimating the number of principal components by one or more of the following criteria, the percentage of explained variance, eigen value-one criterion, the Scree-test and Cross validation. They can be considered as new variables that summarize in an optimal way the variation present in the spectra, in the second step, CLS is applied to the newly obtained latent variables. When co-linearity between original variables occurs, principal component plots often allow better interpretation of the variations observed in the data set than plots of original variables selected by CLS. As modeling method, it is less performant than CLS when performing prediction within the calibration domain and when the model is indeed linear. It is more reliable if extrapolation may be required. It is a linear method, but it is able to perform quite well for moderately nonlinear data. As CLS, it is a global method.

HPLC methods are useful in the determination of drugs in pharmaceutical dosage forms and biological sample. Owing to the widespread use of HPLC in routine analysis.

The purpose of the present study was to investigate the utility of derivative, derivative ratio spectrophotometry, multivariate and HPLC techniques in the assay of metoprolol and felodipine in pharmaceutical preparations without the necessity of sample pretreatment. We also performed a study concerning simultaneous determination of these drugs in human serum samples using HPLC technique.

\section{MATERIALS AND METHODS}

Apparatus and conditions: The Hewlett-Packard liquid chromatographic system consisted of a gradiant Quat pump Model G 1311 A (HP, Avondale, PA, USA) connected with a HP G 1314 A UV-VIS detector (HP, Avondale, PA, USA) operating at $265 \mathrm{~nm}$, a G $1328 \mathrm{~A}$ (Cotati, CA) injection valve, with a $20 \mu$ loop. The chromatographic data were collected and analyzed using HP Chem Station for LC and LC/MS system (Hewlett-Packard, Avondale, PA, USA). The chromatographic separation was performed at ambient temperature $\left(20-22{ }^{\circ} \mathrm{C}\right)$ using an analytical column, Spherisorb ${ }^{\circ}, 5 \mu \mathrm{m}, 4.6$ x $150 \mathrm{~mm}$ i.d., (Waters, Milford, MA, USA). The reversed mobile phase was 
obtained by mixing methanol:water:acetonitrile $(70: 22: 8(\mathrm{v} / \mathrm{v} / \mathrm{v}))$. The flow rate was $0.9 \mathrm{ml} / \mathrm{min}$. Finasteride was used as an internal standard.

- Spectrophotometric measurements were carried out on a computerized Spectronic Gensys 2PC, UV/visible Spectrophotometer (Milton Roy, USA), using $1.00 \mathrm{~cm}$ quartz cells. The obtained spectral data were saved in PC apparatus program and the subsequent statistical manipulation was performed by transferring the spectral data to Microsoft excel XP program and processing them with the standard curve fit package and matrix calculations. Sonicator (Bransonic 220 / BenderHobein), Heater(Salvis) (Heidolph).

-Balance (Mettler Toledo). Curve Expert version 1.37 Copyright ${ }^{\mathcal{O}} 1995-2001$ by Daniel Hyams. GraphPad Instat version 3.05,32 bit for win 95/NT created Sep. 27,2000 Copyright $^{\mathcal{O}} 1992-2000$ by GraphPad software.

Chemicals, reagents and pharmaceutical preparations: All chemicals were of analytical-reagent grade and solvents were of spectrophotometric and HPLC grade. Metoprolol and felodipine and its pharmaceutical dosage form were kindly provided by the Misr Co., Egypt, finasteride used, as internal standard (Aldrich).

HPLC grade methanol and acetonitrile (Merk, Darmstadt, Germany) and doubly distilled water were used for preparing mobile phase solutions. For spectrophotometric studies, methanol [Merk, (Darmstafd, Germany)] was used. Logimax tablets (AstraZeneca Co., Sweden), labeled to contain $5 \mathrm{mg}$ felodipine and $50 \mathrm{mg}$ metoprolol per tablet.

Preparation of standard solutions and calibration: Stock standard solutions containing 0.5 and $5.0 \mathrm{mg}$ metoprolol and felodipine respectively, were dissolved in $10 \mathrm{ml}$ methanol. Standard solutions of both drugs were prepared individually by dilution of the stock solutions with methanol for spectrophotometric methods to obtain concentration range of $5-150 \mu \mathrm{g} / \mathrm{ml}$ for metoprolol and 1-60 $\mu \mathrm{g} / \mathrm{ml}$ for felodipine and in mobile phase preparation for chromatographic method to reach concentration range of $0.15-15$ and $0.03-5$ $\mu \mathrm{g} / \mathrm{ml}$ for metoprolol and felodipine, respectively.

For derivative spectrophotometric method (D1): The values of the D1 amplitudes were measured at $222 \mathrm{~nm}$ (zero-crossing of felodipine) and $235 \mathrm{~nm}$ (zero-crossing of metoprolol) for the determination of metoprolol and felodipine, respectively.
For first derivative of the ratio spectrophotometric method (DD1): According to the theory of the ratiospectra derivative method. The stored UV absorption spectra of standard solutions of metoprolol were divided wavelength-by-wavelength by a standard spectrum of felodipine $(20 \mu \mathrm{g} / \mathrm{ml})$. The first derivative was calculated for the obtained spectra with $\Delta \lambda=4 \mathrm{~nm}$. The amplitudes at $250.1 \mathrm{~nm}$ were measured and found to be linear to the concentrations of metoprolol. For felodipine, the stored UV absorption spectra of standard solutions of felodipine were divided wavelength-bywavelength by a standard spectrum of metoprolol (15 $\mu \mathrm{g} / \mathrm{ml})$. The first derivative was calculated for the obtained spectra with $\Delta \lambda=4 \mathrm{~nm}$. The amplitudes at $224.3 \mathrm{~nm}$ were measured and found to be linear to the concentration of felodipine.

For multivariate method: In order to obtain the calibration matrix for applying CLS and PCR analysis, ten solutions of each of the pure components (metoprolol and felodipine) were prepared in a concentration range of $5-70$ and $1-10 \mu \mathrm{g} / \mathrm{ml}$ for metoprolol and felodipine, respectively. These ranges were previously verified to obey Beer's law for each of the studied drugs in the selected solvent. The absorption data in the range of 200-300 nm (digitized every 1.0 $\mathrm{nm}$ ) were subjected to least squares analysis in order to obtain the calibration $\mathrm{K}$ matrix. Laboratory prepared mixtures were prepared by mixing known amounts of metoprolol with felodipine in different varied proportions in order to verify the precision of the method for analysis of such mixture and matching the commercial tablets with those having comparable concentrations.

For high-performance liquid chromatographic method: Standard solutions were prepared separately with mobile phase by varying concentrations of metoprolol and felodipine in the range $0.15-15$ and $0.03-5 \mu \mathrm{g} / \mathrm{ml}$, respectively. Maintaining concentration of finasteride (IS) at a constant level of $3 \mu \mathrm{g} / \mathrm{ml}$. Triplicate $10 \mu \mathrm{l}$ injections were made for each concentration and peak area ratio of each concentration to the internal standard was plotted against the corresponding concentration to obtain the calibration graph.

Application to pharmaceutical dosage form: For all methods, 10 tablets labeled to contain $5 \mathrm{mg}$ of metoprolol and $50 \mathrm{mg}$ of felodipine were powdered. An 
accurate weight of the powder equivalent to one tablet content was accurately weighed, transferred into a $100 \mathrm{ml}$ calibrated flask, diluted with methanol, stirred for about $10 \mathrm{~min}$ and then completed to volume with the same solvent. This solution was filtered to remove any insoluble matter. After filtration, working solutions were prepared by transferring suitable aliquots of clear filtrate (for HPLC study, adding of the constant amount of internal standard) and diluting with appropriate solvent. The assay was completed as under section 2.3. The concentrations of metoprolol and felodipine were calculated from the corresponding regression equations.

Accuracy study: To study the accuracy of the proposed methods, and to check the interference from excipients present in the dosage form, recovery experiments were carried out by the standard addition method.

This study was performed by addition of different amounts of metoprolol and felodipine to a known concentration of the commercial tablets. The resulting mixtures were analyzed as described under Section 2.3.

Recovery studies in human serum by HPLC method: Serum sample, obtained from healthy individuals (after obtaining their written consent), were stored frozen until assay. After gentle warming, thawing aliquots of serum were spiked with metoprolol and felodipine dissolved in methanol to obtain final concentration of 15 and $5 \mu \mathrm{g} / \mathrm{ml}$, respectively and treated with $500 \mu \mathrm{l}$ acetonitrile as serum protein precipitating agent, then the volume was completed to 2 $\mathrm{ml}$ with the same serum sample. The tubes were vortexed for $5 \mathrm{~min}$ at $1500 \mathrm{~g}$ and then centrifuged for $10 \mathrm{~min}$ at $5000 \mathrm{x} \mathrm{g}$ for getting rid of protein residues. The supernatant was taken carefully, serum samples including various concentrations of metoprolol, felodipine and constant amount of internal standard were injected into the column.

\section{RESULTS AND DISCUSSION}

D1 method: As shown in Fig. 1, the zero-order spectra of standard drugs were found to be overlapped making their simultaneous determination difficult. In contrast, the D1 spectrum of each pure drug was found to show zero-crossing points (Fig. 2) and assisted in their simultaneous estimation. In practice, the wavelength selected is that which exhibits the best linear response, giving a zero or near zero intercept on the coordinate of the calibration graph, and not affected by the presence of any other component. The shape of the first derivative spectra is adequate for determining metoprolol in the presence of felodipine and vice versa. Metoprolol was determined by measuring of its D1 amplitude at the zero-crossing point of felodipine (at $222 \mathrm{~nm}$ ). While felodipine was determined by measuring of its D1 at the zero-crossing point of metoprolol (at $235 \mathrm{~nm}$ ). Linear relationships between derivative amplitude and drug concentration were obtained over the concentration range $20-150 \mu \mathrm{g} / \mathrm{ml}$ for metoprolol and 10-60 $\mu \mathrm{g} / \mathrm{ml}$ for felodipine. The linear regression equations together with correlation coefficients slope and intercept, R.S.D. of slope and intercept repeatability (within day) and reproducibility (between-day) obtained for each drug are collected in Table 1.

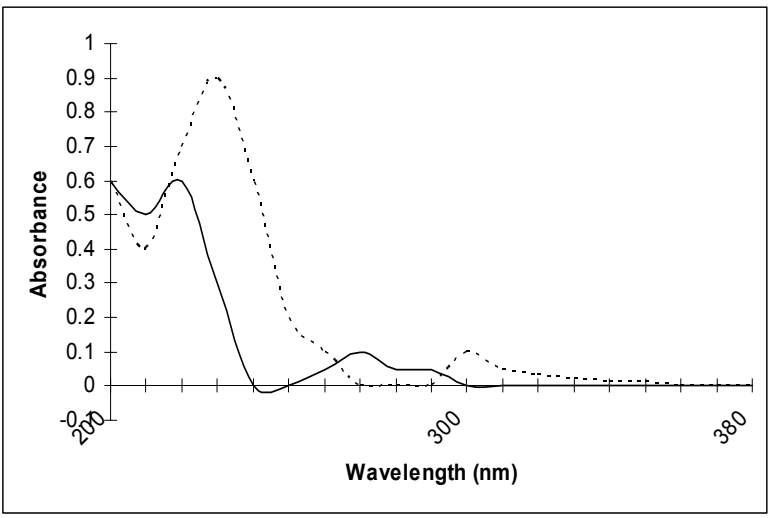

Fig 1: Absorption spectra of metoprolol (-), $50 \mu \mathrm{g} / \mathrm{ml}$ and felodipine (……), $25 \mu \mathrm{g} / \mathrm{ml}$.

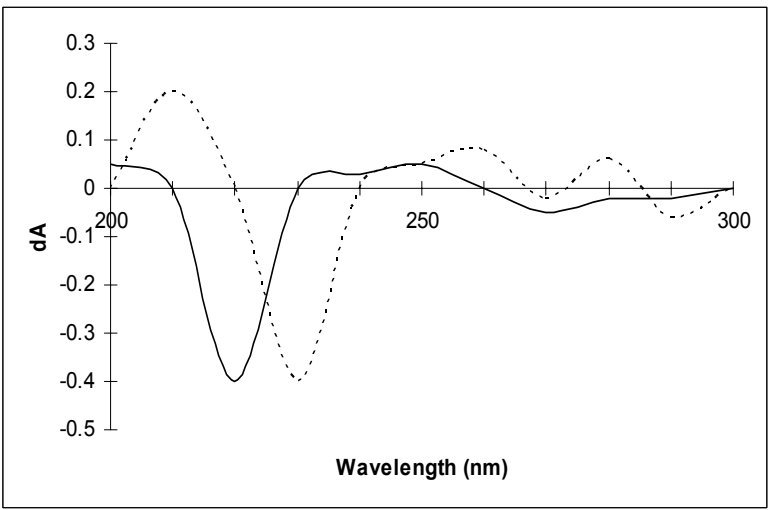

Fig. 2 :First derivative zero-crossing determination of metoprolol (-) at $222 \mathrm{~nm}$ and felodipine $(\cdots \cdots)$ at $235 \mathrm{~nm}$

DD1 method: The influence of $\Delta \lambda$ for obtaining the first derivative of the ratio spectra as well as, the effect of divisor concentration on the calibration graphs for the proposed mixture was studied in order to select the best factors affecting the determination. Results 
indicated that $\Delta \lambda=4 \mathrm{~nm}$ was most suitable one, while the divisor concentration has no significant effect on the assay results for the studied mixtures.

For determination of metoprolol, the absorption spectra of metoprolol were divided by that of standard solutions of felodipine $(20 \mu \mathrm{g} / \mathrm{mL})$ and the absorption spectra of felodipine were divided by that of standard solutions of metoprolol $(8 \mu \mathrm{g} / \mathrm{mL})$, figures 3 and 4 , respectively.

The first derivative of the developed ratio spectra were calculated with $\Delta \lambda=4 \mathrm{~nm}$. Figures 5 and 6 show that, metoprolol can be determined by measuring the amplitude at many wavelengths where felodipine has no contribution, but it was found that the amplitude at $250.1 \mathrm{~nm}$ give the most accurate and sensitive results (Figure 5). Figure 6, shows that felodipine can be determined by measuring the amplitude at many wavelengths where metoprolol have no contribution, but it was found that the amplitude at $224.3 \mathrm{~nm}$ give the most accurate and sensitive results.

The proposed method is applicable over the range 20$150 \mu \mathrm{g} / \mathrm{ml}$ for metoprolol and $10-60 \mu \mathrm{g} / \mathrm{ml}$ for felodipine. The characteristic parameters and necessary statistical data of the regression equations, LOD and LOQ values, respectively and reproducibility data are collected in Table 1.

Repeatability and reproducibility variables were characterized by R.S.D.(\%) and by the difference between theoretical and measured concentrations. There was no significant difference for the assay, which was tested within-day (repeatability) and between-days (reproducibility).

In order to demonstrate the validity and applicability of the proposed DD1 method, recovery studies were performed by analyzing laboratory prepared mixtures of metoprolol and felodipine with different composition ratio (Table 2).

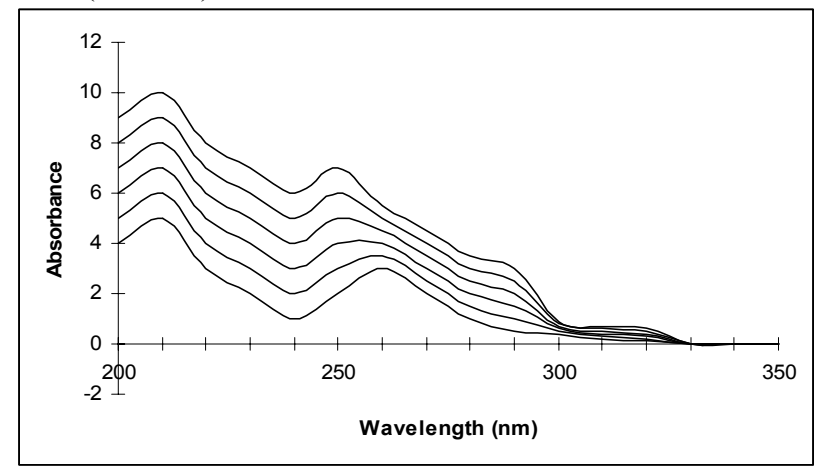

Fig. 3: Ratio spectra of metoprolol ( $20-150 \mu \mathrm{g} / \mathrm{mL}$ ). Divisor is $20 \mu \mathrm{g} / \mathrm{ml}$ felodipine

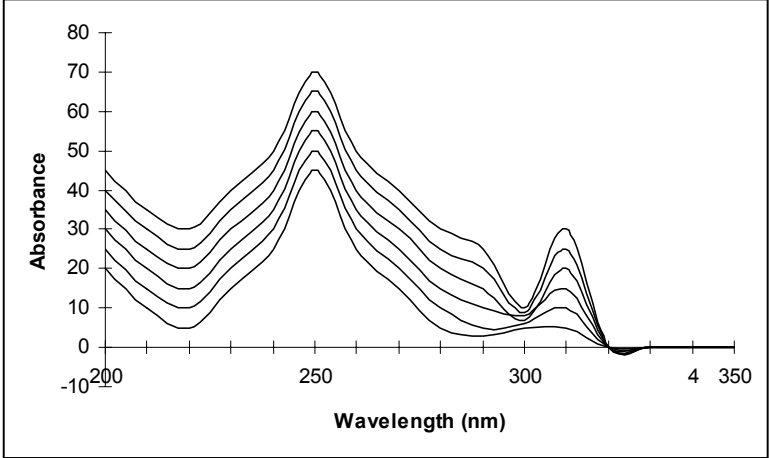

Fig. 4: Ratio spectra of felodipine $(10-60 \mu \mathrm{g} / \mathrm{mL})$. Divisor is $8 \mu \mathrm{g} / \mathrm{ml}$ metoprolol

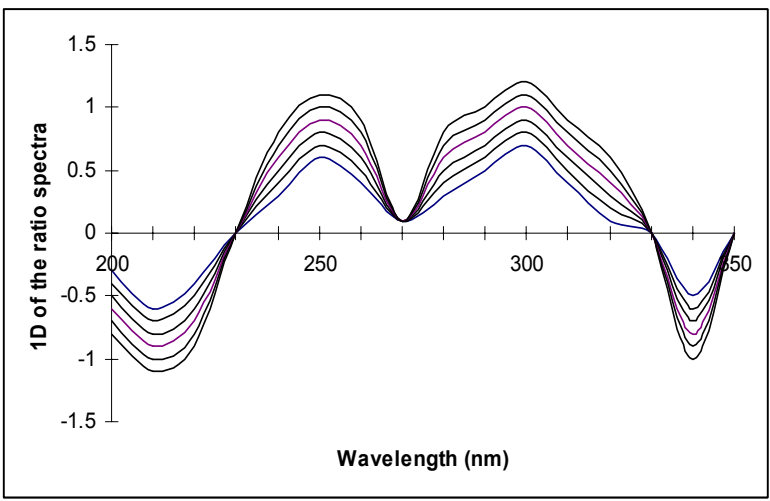

Fig. 5: First derivative of the ratio spectra metoprolol ( $20-150 \mu \mathrm{g} / \mathrm{mL}$ ). Divisor is $20 \mu \mathrm{g} / \mathrm{ml}$ felodipine

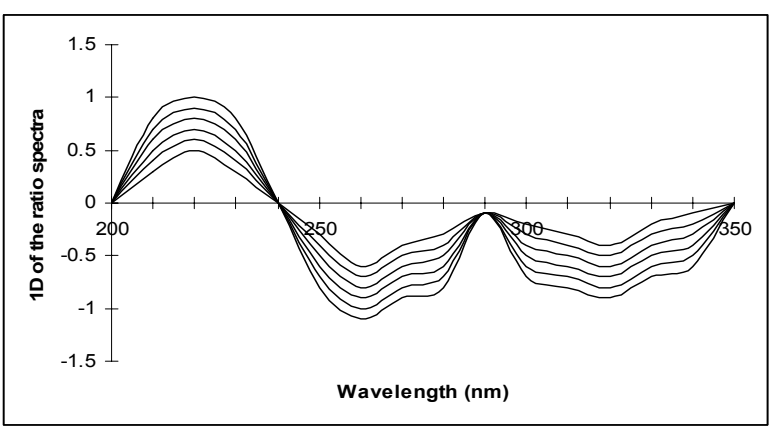

Fig. 6: First derivative of the ratio spectra of felodipine (10-60 $\mu \mathrm{g} / \mathrm{mL})$. Divisor is $8 \mu \mathrm{g} / \mathrm{ml}$ metoprolol

Multivariate method: The absorption spectra of the studied drugs are shown in Figure 1. As could be seen, a considerable degree of spectral overlapping occurs in the region from 200 to $300 \mathrm{~nm}$ for metoprolol and felodipine. The degree of spectral overlapping was given by $\left(\mathrm{D}_{\mathrm{i}}\right)^{0.5}$. In case of the presently studied compounds, the spectra lead to $\mathrm{D}_{\mathrm{i}}=0.50$ implying a $88.21 \%$. 
Am. J. Applied Sci., 4 (9): 709-717, 2007

Table 1: Statistical data for the calibration graphs of metoprolol and felodipine by 1D, 1DD, CLS, PCR and HPLC methods

\begin{tabular}{lllllllllll}
\hline & \multicolumn{2}{c}{ Metoprolol } & \multicolumn{3}{c}{ Felodipine } & & \\
& D1 & DD1 & CLS & PCR & HPLC & D1 & DD1 & CLS & PCR & HPLC \\
\hline Linearity range $(\mu \mathrm{g} / \mathrm{ml})$ & $20-150$ & $20-150$ & $10-70$ & $5-35$ & $0.15-15$ & $10-60$ & $10-60$ & $2-10$ & $1-6$ & $0.03-5$ \\
Slope & 0.0132 & 0.0084 & 0.9866 & 0.7506 & 0.2179 & 0.0065 & 0.0065 & 0.8433 & 0.8438 & 0.1959 \\
Intercept & 0.008 & 0.005 & -0.001 & 0.014 & 0.001 & 0.022 & 0.009 & 0.003 & 0.019 & 0.002 \\
Correlation coefficient & 0.9998 & 0.9997 & 1.0000 & 0.9999 & 0.9999 & 0.9997 & 0.9999 & 0.9999 & 0.9999 & 0.9999 \\
R.S.D. of the slope & 0.57 & 0.65 & 0.65 & 0.49 & 0.75 & 0.45 & 0.77 & 0.64 & 0.76 & 0.66 \\
R.S.D. of the intercept0.09 & 0.07 & 0.08 & 0.15 & 0.07 & 0.12 & 0.42 & 0.32 & 0.23 & 0.19 & \\
LOD $(\mu \mathrm{g} / \mathrm{ml})$ & 5.44 & 5.70 & 4.75 & 5.79 & 3.75 & 9.72 & 3.43 & 6.44 & 8.64 & 2.50 \\
LOQ $(\mu \mathrm{g} / \mathrm{ml})$ & 15.34 & 12.65 & 13.05 & 15.86 & 10.08 & 40.64 & 7.54 & 19.54 & 24.90 & 4.32 \\
Repeatability (R.S.D.; \%) & 0.94 & 0.45 & 0.94 & 0.84 & 0.59 & 0.43 & 0.84 & 0.69 & 0.59 & 0.69 \\
Reproducibility (R.S.D.; \%) & 0.99 & 0.59 & 0.59 & 0.93 & 0.89 & 0.85 & 0.89 & 0.85 & 0.85 & 0.57 \\
\hline
\end{tabular}

Table 2: Determination of metoprolol and felodioine in authentic, laboratory prepared mixtures and pharmaceutical dosage form using D1, DD1, CLS, PCR, HPLC and reported methods.

\begin{tabular}{|c|c|c|c|c|c|c|c|c|}
\hline Standard solution & Reported & D1 & & DD1 & & CLS & PCR & HPLC \\
\hline \multirow[t]{3}{*}{ Authentic metoprolol } & $99.4 \pm 0.25[32]$ & $99.2 \pm 0.40$ & & $99.3 \pm 0.33$ & & $99.1 \pm 0.40$ & $99.7 \pm 0.49$ & $99.3 \pm 0.44$ \\
\hline & & $\mathrm{t}=1.24$ & & $\mathrm{t}=1.07$ & & $\mathrm{t}=1.99$ & $\mathrm{t}=1.24$ & $t=0.71$ \\
\hline & & $\mathrm{F}=4.00$ & & $\mathrm{~F}=1.83$ & & $\mathrm{~F}=2.67$ & $\mathrm{~F}=4.00$ & $F=3.16$ \\
\hline \multirow[t]{5}{*}{ Authentic felodipine } & $100.2 \pm 1.37[32]$ & $99.7 \pm 0.99$ & & $100.3 \pm 1.01$ & $100.1 \pm 0.98$ & $99.7 \pm 0.69$ & $100.1 \pm 0.87$ & \\
\hline & & 1.88 & & 0.98 & & 1.00 & 0.96 & \\
\hline & & 0.48 & & 0.76 & & $t=0.72$ & $\mathrm{t}=0.14$ & $t=0.15$ \\
\hline & & $t=0.77$ & & $\mathrm{t}=0.15$ & & & & \\
\hline & & $F=1.92$ & & $\mathrm{~F}=1.88$ & & $\mathrm{~F}=2.47$ & $F=3.92$ & $\mathrm{~F}=2.47$ \\
\hline \multirow{2}{*}{\multicolumn{2}{|c|}{ Laboratory prepared mix.for metoprolol $100.1 \pm 0.74$}} & $100.0 \pm 0.85$ & & $99.9 \pm 1.40$ & & $100.1 \pm 1.39$ & $100.3 \pm 1.42$ & \\
\hline & & 0.86 & & 0.92 & & 1.18 & 1.18 & 1.19 \\
\hline \multirow{2}{*}{\multicolumn{2}{|c|}{ Laboratory prepared mix.for felodipin }} & $99.5 \pm 0.45$ & & $99.4 \pm 0.42$ & & $99.7 \pm 0.39$ & $99.7 \pm 0.40$ & $99.7 \pm 0.30$ \\
\hline & & 0.20 & & 0.18 & & 0.15 & 0.16 & 0.09 \\
\hline \multirow{2}{*}{\multicolumn{2}{|c|}{ Logimax tab. for metoprolol }} & $100.2 \pm 0.65$ & & $99.2 \pm 0.66$ & & $99.8 \pm 0.69$ & $99.9 \pm 0.49$ & $99.4 \pm 0.66$ \\
\hline & & 0.42 & & 0.44 & & 0.48 & 0.24 & 0.66 \\
\hline \multirow{2}{*}{\multicolumn{2}{|c|}{ Logimax tab. for felodipine }} & $99.9 \pm 0.80$ & & $99.7 \pm 0.59$ & & $99.7 \pm 0.90$ & $99.6 \pm 0.84$ & $99.8 \pm 0.60$ \\
\hline & & 0.64 & & 0.59 & & 0.81 & 0.71 & 0.36 \\
\hline \multirow{2}{*}{\multicolumn{2}{|c|}{ Standard addition technique for metoprolol $\quad 100.2 \pm 0.68$}} & $99.47 \pm 0.54$ & $99.49 \pm 0.52$ & $99.48 \pm 0.52$ & $99.7 \pm 0.35$ & & & \\
\hline & & 0.46 & & 0.29 & & 0.27 & 0.27 & 0.12 \\
\hline \multirow{2}{*}{\multicolumn{2}{|c|}{ Standard addition technique for felodipine }} & $99.3 \pm 0.33$ & & $99.4 \pm 0.30$ & & $99.5 \pm 0.29$ & $99.2 \pm 0.31$ & $99.6 \pm 0.35$ \\
\hline & & 0.11 & & 0.09 & & 0.08 & 0.10 & 0.12 \\
\hline
\end{tabular}

Theoretical values at $95 \%$ confidence limit are $\mathrm{t}=3.58$ and $\mathrm{F}=4.28$

Table 3: Results obtained for metoprolol and felodipine analysis from human serum using HPLC method

\begin{tabular}{|c|c|c|c|c|}
\hline & \multicolumn{2}{|c|}{ Metoprolol } & \multicolumn{2}{|c|}{ Felodipine } \\
\hline Added $(\mu \mathrm{g} / \mathrm{ml})$ & 2.00 & 10.00 & 10.00 & 30.00 \\
\hline $\mathrm{n}$ & 4 & 4 & 4 & 4 \\
\hline Found $(\mu \mathrm{g} / \mathrm{ml})$ & 1.98 & 9.81 & 9.78 & 29.38 \\
\hline R.S.D. (\%) & 1.04 & 0.7 & 0.84 & 0.94 \\
\hline Average recovery $(\%)$ & 99.20 & 98.07 & 97.80 & 97.93 \\
\hline
\end{tabular}

Several laboratory prepared mixtures were subjected to the CLS and PCR analysis in order to confirm the suitability of the calibration model for determination of the studied drugs in the pharmaceutical sample solutions.

The results of commercial dosage form and laboratory prepared mixtures analysis with comparable concentrations were found closely matched. This indicated that, excipients and additives did not interfere with the determinations. Moreover, the results of dosage form analysis were compared with those obtained by applying reported methods. As shown in
Table 2, the results are in good agreement with those of the reported procedures as indicated by the calculated $t$ and $\mathrm{F}$ values.

High-performance liquid chromatographic method: Drug concentration is monitored during various phases of pharmaceutical development, such as formulation and stability studies, quality control and pharmacological testing in animals and humans. All these investigations require reliable and validated analytical methods in order to measure drugs in pharmaceutical formulations and biological samples. 
In order to perform the simultaneous elution of metoprolol and felodipine peaks under gradient conditions, the mixtures of methanol, acetonitrile and water in different combinations at various flow rates were assayed. The optimum wavelength for detection was $260 \mathrm{~nm}$ at which much better detector responses for both drugs were obtained. The mixture of methanol: acetonitrile: water $(70: 22: 8(\mathrm{v} / \mathrm{v} / \mathrm{v}))$ at $0.9 \mathrm{ml} / \mathrm{min}$ flow rate, proved to be better than the other mixtures and flow rates for separation, since the chromatographic peaks were better defined, resolved and free from tailing. The retention times were $2.06 \mathrm{~min}$ for metoprolol, $3.36 \mathrm{~min}$ for felodipine and $4.32 \mathrm{~min}$ for finasteride (IS).

Finasteride was chosen as the internal standard because it showed a shorter retention time with better peak shapes and better resolution, compared to other potential internal standards.

Resolution and selectivity factors for this system were found 2.22 and 2.51 for metoprolol and felodipine, respectively. Tailing and capacity factors were obtained as 1.21 and 0.92 for metoprolol and 1.51 and 1.55 for felodipine. The variation in retention times among six replicate injections of metoprolol and felodipine standard solutions was very low, rendering a R.S.D of 0.90 and $0.71 \%$, respectively. The results obtained from system suitability tests are in agreement with the USP requirements.

Peak area ratios (Asample $\backslash$ AIS) were plotted against corresponding concentrations in the range of $0.15-15$ $\mu \mathrm{g} / \mathrm{ml}$ for metoprolol and $0.03-5 \mu \mathrm{g} / \mathrm{ml}$ for felodipine. Linear regression parameters of the peak area rations versus concentrations of metoprolol and felodipine are presented in Table 1 . The results showed highly reproducible calibration curves with correlation coefficients $>$ o.999. Statistical data of the regression equations are shown in Table 1. The LOD and LOQ values were calculated as described in Section 3.1.

In order to demonstrate the validity and applicability of the proposed HPLC method, recovery tests were carried out by analyzing laboratory prepared mixtures of metoprolol and felodipine, with different ratios (Table 2).

Application to pharmaceutical dosage form: The four proposed methods are successfully applied for the simultaneous determination of both drugs in Logimax tablets ${ }^{\circledR}$ without interference of the excipients present and without prior separation. The utility of the four proposed methods was verified by replicate estimations of the pharmaceutical preparation and results obtained are evaluated statistically (Table 2).
Validity of the proposed methods: Statistical analysis of the results obtained for the proposed methods (Table 2 ), shows that all the suggested measurements are equally precise and accurate to the reported methods [32].

Application to the serum samples: In order to check the applicability of the proposed HPLC method to biological materials, the recovery studies were performed on human serum samples (Table 3). Analysis of drugs in serum by HPLC technique always suffers from being tedious and expensive. In our proposed method, simple extraction procedure was applied using acetonitrile, then after centrifugation supernatant is directly injected and analyzed.

A typical chromatogram was obtained for the serum spiked with metoprolol and felodipine, showing no interference from endogenous substances present in serum.

\section{CONCLUSION}

The D1and DD1, multivariate (CLS and PCR) and HPLC methods enable the quantitation of metoprolol and felodipine binary mixture with good accuracy and precision, either in laboratory prepared samples or in pharmaceutical dosage forms. By comparing the results of the four proposed methods, no significant difference is obtained; HPLC method has the superiority over spectrophotometric methods in analyzing the binary mixture of studied drugs. All of the proposed procedures have the advantage of being rapid, precise and direct. DD1 method has two advantages over the D1 method, the first is the high sensitivity and accuracy, the second is the easy measurements at any peak signal giving higher values. Classical least squares and PCR multivariate calibration analysis were developed for the analysis of the laboratory prepared mixtures and commercial tablets. The good recoveries obtained in all cases as well as the reliable agreement with the reported procedures proved that, the proposed method could be applied efficiently for determination of metoprolol and felodipine binary mixture with quite satisfactory precision and could be easily used in a quality control laboratory for their analysis. The proposed HPLC method was used as a versatile reference method and it is suitable for the analysis of metoprolol and felodipine in biological fluid. Good resolution between the studied drugs and the chosen internal standard was obtained in a short analysis time usin simple extraction procedure without interference of endogenous substances present in serum samples. 


\section{REFERENCES}

1. El-Hawary M. B., khayall M. T. and Isaak Z., 1985. Hand Book of Pharmacology, The scientific Book Center, S.O.P. Press, and Cairo.

2. Vojko K. and Robert R., 2003. HPLC determination of tramadol in human breast milk . J. Pharm. Biomed. Anal., 32: (4-5), 1061-1066.

3. Ney Carter C. B., Gustavo D. M., Rafae E. B. 1, Paulo G. , Celso H. O. and Gilberto D. N., 2005. Verapamil quantification in human plasma by liquid chromatography coupled to tandem mass spectrometry: An application for bioequivalence study J. Chromatogr. B, 827: (2, 5),165-172.

4. Agrawal Y. K. and Patel R. N., 2005. Chiral chromatographic separation of $\beta$-blockers. J. Chromatogr. B, 820: (1), 23-31.

5. Niina M., Tuula T. and Leif K., 2006. Analysis of neutral and basic pharmaceuticals in sewage treatment plants and in recipient rivers using solid phase extraction and liquid chromatographytandem mass spectrometry detection J. Chromatogr. A,1134: (1-2, 17), 101-111.

6. Maguregui M. I., Alonso R. M. and Jimenez R. M., $\quad 1995 . \quad$ High-performance liquid chromatography with amperometric detection applied to the screening of $\beta$-blockers in human urine J. Chromatogr. B, 674: (1),85-91.

7. Hassan S. M., Abou-Sekkina M. M., El-Ries M. A. and Wassel A. A., 2003. Polymeric matrix membrane sensors for sensitive potentiometric determination of some $\beta$-blockers in pharmaceutical preparations. J. Pharm. Biomed. Anal., 32 : (1), 175-180 .

8. Penny D. C., Glen B. B. and Ronald T. C., 1997. $A$ rapid and sensitive electron-capture gas chromatographic procedure for analysis of metoprolol in rat brain and heart. J. Pharm. Toxico., 38: (1), 27-32.

9. Olof S., Mats J. and Frans W. L., 2000. The synthesis of metoprolol monitored using Raman spectroscopy and chemometrics. Euro. J. Pharm.1 Sci., 11: (2), 141-155.

10. Vera L. L., Pierina S. B., Paula M. C., and Valéria A. P., 2000. Enantioselective analysis of metoprolol in plasma using high-performance liquid chromatographic direct and indirect separations: applications in pharmacokinetics. Evandro J. Chromatogr. B: Biomed. Sci. Appl., 738: (1), 27-37.
11. Salem H., 2002. Spectrophotometric determination of beta-adrenergic blocking agents in pharmaceutical formulations. J. Pharm. Biomed. Anal., 29: (3), 527-538.

12. David B., Nicklas A. P., Timothy R. G. and Frank J. L., 2005. Occurrence and fate of pharmaceutically active compounds in the environment, a case study: Höje River in Sweden J. Hazard. Mate., 122: (3), 195-204.

13. Lazaros A. N., Indu P., P. Pratik, M. Megan, S. You-Tang and P. S. Richard, 2006. The Effects of Combined Versus Selective Adrenergic Blockade on Left Ventricular and Systemic Hemodynamics, Myocardial Substrate Preference, and Regional Perfusion in Conscious Dogs With Dilated Cardiomyopathy J. Ameri. Coll. Cardiolo., 47: (9), 1871-1881.

14. A.López J., Martínez V., Alonso R. M. and Jiménez R. M., 2000. High-performance liquid chromatography with amperometric detection applied to the screening of 1,4-dihydropyridines in human plasma J. Chromatogr. A, 870: (1-2), 105114.

15. Katja H. and Franz B., 2002. Application of column-switching liquid chromatography-tandem mass spectrometry for the determination of pharmaceutical compounds in tissue samples $\mathrm{J}$. Chromatogr. B, 769: (1), 9-26.

16. Ana B. B., Rosa M. J. and Rosa M. A., 2004. Simultaneous determination of five 1,4dihydropyridines in pharmaceutical formulations by high-performance liquid chromatographyamperometric detection J. Chromatogr., 1031:1-2, 275-280.

17. Gottfries J., Ahlbom J., Harang V., Johansson E., Josefson M., T., Pettersson A. and Torstensson A. A, 1994. Validation of an extended release tablet dissolution testing system using design and multivariate analysis Int. J. Pharm.,106: (2),141148 .

18. Marciniec B., Jaroszkiewicz E. and Ogrodowczyk M., 2002. The effect of ionizing radiation on some derivatives of 1,4-dihydropyridine in the solid state. Int. J. Pharm., 233: (1-2), 207-215.

19. Basavaiah K., Chandrashekar U. and Prameela H. C., 2003. Sensitive spectrophotome-tric determination of amlodipine and felodipine using iron(III) and ferricyanide. Farmaco II, 58: (2), 141148 .

20. Salem H., 1999. Derivative Spectrophotom-etric Determination of Two Component Mixtures. Chin. Pharm. J., 51: 123-142 
21. Mabrouk M. M., Salem H., Radwan M. F. and Kaood T. S., 2003. Derivative Spectrophoto-metric Determination of Some Selected Antihypertensive Combinations. Egypt J. Biomed. Sci., 12: 141-173.

22. Salem H., 2006. Atomic Absorption Spectrometric, Spectrophotometric and Derivative Spectrophotometric Determi-nation of Sildenafil Citrate (Viagra) in Bulk Powder and in Pharmaceutical Dosage Form.J. J. Appl. Sci., 8: (1), 28-43.

23. Moor D., Valette G., Darque A., Zho X. J. and Sommadossi J. P., 2002. Validated spectrophotometric analysis of certain binary mixtures. J. Am. Soc. Mass Spectrom. 11: 1134.

24. Kenney K. B., Wring S. A., Carr R. M., Well G. N. and Dunn J. A., 2000. Utility of derivative ratio spectrophotometric analysis of selected drugs. J. Pharm. Biomed. Anal., 22: 967.

25. Shamsipur M. and Jalali F., 2000. Spectrophotometric determination of two components mixtures. Anal. Sci., 16: 5, 549-555.

26. Sheng Q., Peng T. Z., Hu X. B. and Yang L. G., 1998. Selective spectrophotometric determination of certain drugs. Fensi Hauxa, 26:11, 1315-1318.
27. Mushik O. V., Tkach V. I., Karanadeva S. M., Glukhoya M. J. and Tsyganpk L. P., 1998. Analytical study of certain drugs. J. Anal. Chem., 53:1110-1112.

28. Mohamed H., Mohamed R. , Ebrahim G. and Karim A. , 2005. Utility of multivariate analysis of certain drugs. FARMACO, 60: 451-458.

29. Abde El-Maaboud I. and Pakinaz Y., 2002. Chemometric assisted analysis of paracetamol in presence of certain drugs. J. Pharm. Sci., 5-6: 1122.

30. Jose A., Aurelia A. and Pablo F., 2001.Spectrophotometric determination of selected drugs in their pharmaceutical dosage forms. Analytica Chimica Acta, 449: 179-187.

31. Hector C. and Alejandro C., 1998. Utility of CLS and PCR for analysis of certain drugs. Talanta 47:103-108.

32. Moffat A. C., Jackson J. V., Moss M. S., Widdop B., Greefield E. S., 1986. Clarke's Isolation and Identification of Drugs, $2^{\text {nd }}$ ed., The pharmaceutical press, London.

33. Bartos M. J., 1974. Colourimetric and Fluorimetric Analysis of Organic Compounds and Drugs, Marcel Dekker, New York, 623-630. 